\title{
Torque ripple minimization for a Switched Reluctance Motor
}

\author{
Balduí Blanqué ${ }^{1}$, Arnau Dòria-Cerezo ${ }^{1,2}$ \\ ${ }^{1}$ Dept. Electrical Egineering \\ ${ }^{2}$ Inst. of Industrial and Control Engineering \\ Universitat Politècnica de Catalunya \\ Vilanova i la Geltrú, Spain \\ blanque@ee.upc.edu, arnau.doria@upc.edu
}

\section{Acknowledgments}

B. Blanqué and A. Dòria-Cerezo were partially supported by the Spanish government research projects DPI2006-09880 and DPI2007-62582, respectively.

\section{Keywords}

$<<$ Switched reluctance drive $>>$ and $<<$ Control methods for electrical systems $>>$.

\begin{abstract}
This paper presents the desired current references in order to minimize the torque ripple for a Switched Reluctance Motor. From the torque equation, and using switch on and switch off angles, the desired current waveforms are obtained. Simulations validates the study and are compared with the constant current references case.
\end{abstract}

\section{Introduction}

Recently, switched reluctance machines (SRM) have emerged as a popular alternative to brushless DC and $\mathrm{AC}$ motors because of its simple and reliable structure, i.e., no magnets and windings on the rotor [5]. One of the main advantages of the SRM is that it can produce high motor torque at low speeds. SRM are present in many home applications (washing machines, door actuators), robotics, automotive applications and, recently, in hybrid electrical vehicles (see [6] or [3]). However, it is not yet widely accepted due to the non-linear nature of its torque production, which causes torque ripples and thereby vibrations and noise.

Figure 1 shows a cross section scheme of one of the most typical configurations, the $6 / 4$ switched reluctance machine. The different number of poles for the stator and rotor, 6 and 4 respectively, allows to start and operate in both directions.

The SRM requires an specific drive circuit which supplies each phase coil with a switched voltage. This power electronic supply plays an important role in the torque production, since each phase must be switched on and turned off depending on the position. Figure 2 displays the SRM and its associated classic power converter. Notice that the knowledge of the rotor position is required to generate the appropriate voltages.

Torque ripple is a problem already treated in the literature. Studies of torque ripple minimization can be found in [1], [2] and [4], or more recently in [7]. In this paper, a mathematical analysis of the ideal currents waveform have been done. From the SRM torque equation, a suitable current profiles are obtained in order to ensure an ideal torque tracking.

This paper is organized as follows. In the first Section, the dynamical model of the SRM is presented. Then, in the following Section, the switch on and switch off angles are defined, and the desired phase currents are computed in order to obtain a the desired torque waveform. Simulations of the proposed methodology are also presented and, finally, conclusions are stated in the last Section. 


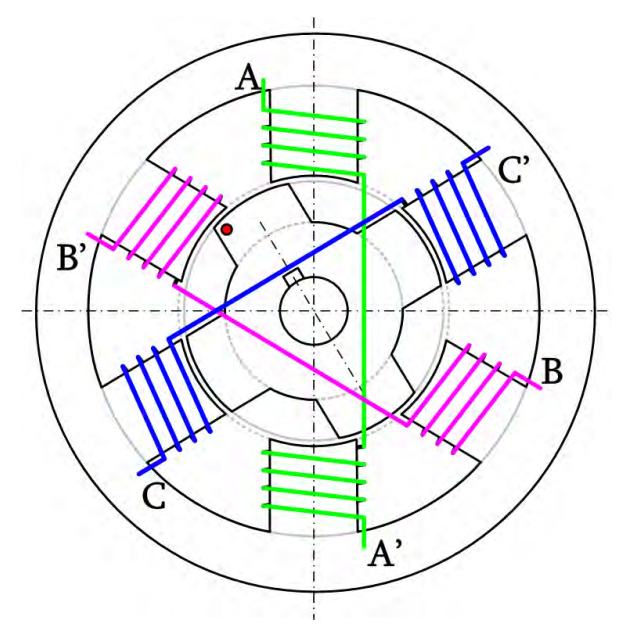

Figure 1: Scheme of a cross section for a $6 / 4$ switched reluctance machine.

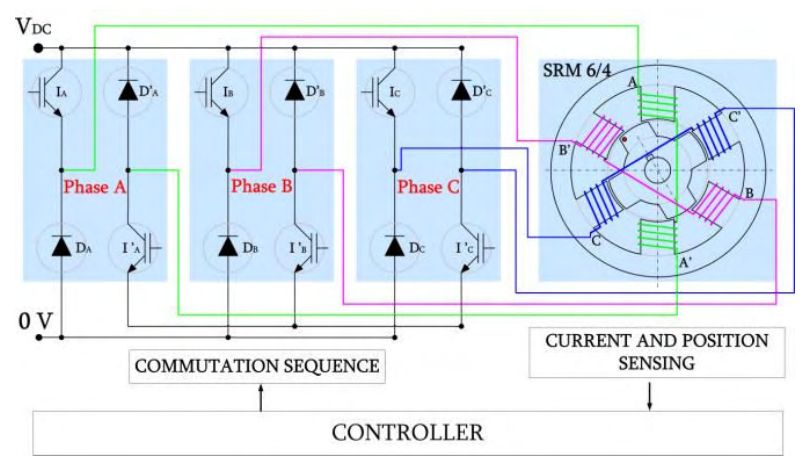

Figure 2: Driver scheme for a 6/4 switched reluctance machine.

\section{The switched reluctance machine model}

In this section the dynamical model of a SRM is introduced. First, the electrical part of the system is described and from the magnetic energy function, the electrical torque is defined. Then the whole SRM dynamics is completed by the mechanical part. The presented model is presented as a generalized form, and then the most accepted assumptions are used in order to obtain an specific model to study.

The electrical equations are given by

$$
V=R i+\dot{\lambda}
$$

where $V \in \mathbb{R}^{n}$ are the voltages applied to the stator windings, $i \in \mathbb{R}^{n}$ are the stator currents, $\lambda \in \mathbb{R}^{n}$ are the fluxes, $R=\operatorname{diag}\left\{r_{1}, r_{2}, \ldots, r_{n}\right\} \in \mathbb{R}^{n \times n}$ is the resistance matrix (where $r$ is the resistance value of each stator winding) and $n$ denotes the number of stator phases.

The relationship between the energy and co-energy variables is described by

$$
\lambda=\mathcal{L} i
$$

where $\mathcal{L}$ is the inductances matrix, which is symmetric and positive definite $\mathcal{L}=\mathcal{L}^{T}>0$. The magnetic energy is given by

$$
H_{E M}=\frac{1}{2} \lambda^{T} \mathcal{L}^{-1} \lambda
$$

and the mechanical equation is

$$
\tau_{L}=J \dot{\omega}+b \omega+\tau_{e}
$$


where $\omega$ is the mechanical speed, $\tau_{L}$ is an external torque, $\tau_{e}$ is the electrical torque, $J$ is the rotor inertia, and $b$ represents the viscous damping. The electrical torque $\tau_{e}$ is produced by the interaction of the magnetic fields, i.e. it can be written from the magnetic energy (3), as ${ }^{1}$

$$
\tau_{e}=\partial_{\theta} H_{E M}
$$

or

$$
\tau_{e}=\frac{1}{2} \lambda^{T} \partial_{\theta}\left(\mathcal{L}^{-1}\right) \lambda
$$

Tacking into account $\partial_{\theta}\left(\mathcal{L}^{-1}\right)=\left(\mathcal{L}^{-1}\right)^{T} \cdot \partial_{\theta} \mathcal{L} \cdot \mathcal{L}^{-1}$ and (2), the torque can be expressed in terms of currents as

$$
\tau_{e}=-\frac{1}{2} i^{T} \partial_{\theta} L i
$$

Summarizing, the most important part of the model is how the inductance matrix, $L$, is defined. Including saturation effects implies $\mathcal{L}=\mathcal{L}(i)$, and phase magnetic coupling can be also modelled introducing cross terms in $\mathcal{L}$. In order to show the proposed methodology, let us to use the widely accepted assumption that the stator phases are magnetically decoupled [5], i.e. the mutual inductances are neglected, which implies

$$
\mathcal{L}=\left[\begin{array}{cccc}
L_{1} & 0 & \ldots & 0 \\
0 & L_{2} & \ldots & 0 \\
\vdots & \vdots & \ddots & \vdots \\
0 & 0 & \ldots & L_{n}
\end{array}\right]
$$

Also, in a first approximation, it is usual to describe each phase inductance function as a Fourier series expansion and keep then only the first harmonic component. Then,

$$
L_{j}=l_{0}-l_{1} \cos \left(N_{r} \theta+(j-1) \frac{2 \pi}{n}\right)
$$

where $j=1, \ldots, n, \theta$ is the rotor position, $N_{r}$ is the number of rotor poles, and $l_{0}$ and $l_{1}$ are constants corresponding to the zero and the first harmonic components. In this case, the $l_{0}$ and $l_{1}$ inductance constants corresponds to the inductance value at the alignment and non-alignment positions, respectively.

With these assumptions $\partial_{\theta} \mathcal{L}$ yields

$$
\partial_{\theta} \mathcal{L}=\left[\begin{array}{cccc}
\partial_{\theta} L_{1} & 0 & \ldots & 0 \\
0 & \partial_{\theta} L_{2} & \ldots & 0 \\
\vdots & \vdots & \ddots & \vdots \\
0 & 0 & \ldots & \partial_{\theta} L_{n}
\end{array}\right]
$$

where

$$
\partial_{\theta} L_{j}=l_{1} N_{r} \sin \left(N_{r} \theta+(j-1) \frac{2 \pi}{n}\right) .
$$

And finally, the torque can be expressed as

$$
\tau_{e}=\frac{1}{2} \sum_{j=1}^{n}-\partial_{\theta} L_{j} i_{j}^{2}
$$

or, using (11),

$$
\tau_{e}=-\frac{1}{2} l_{1} N_{r} \sum_{j=1}^{n} \sin \left(N_{r} \theta+(j-1) \frac{2 \pi}{n}\right) i_{j}^{2} .
$$

\section{Torque ripple minimization via tracking currents}

Usually, the torque ripple minimization is done using the torque-angle-current characteristics [5]. Other methods can be found in [1], where authors proposes a PWM strategy with the current profiles.

Figure 3 shows the proposed control scheme. The current references block generate the ideal current in order to achieve the desired torque. Then an inner current loop is required to guarantee the current tracking for each phase.

\footnotetext{
${ }^{1}$ In this paper, to simplify the notation, the $\frac{\partial}{\partial \theta} f(\theta)$ operations has been also defined as $\partial_{\theta} f(\theta)$.
} 


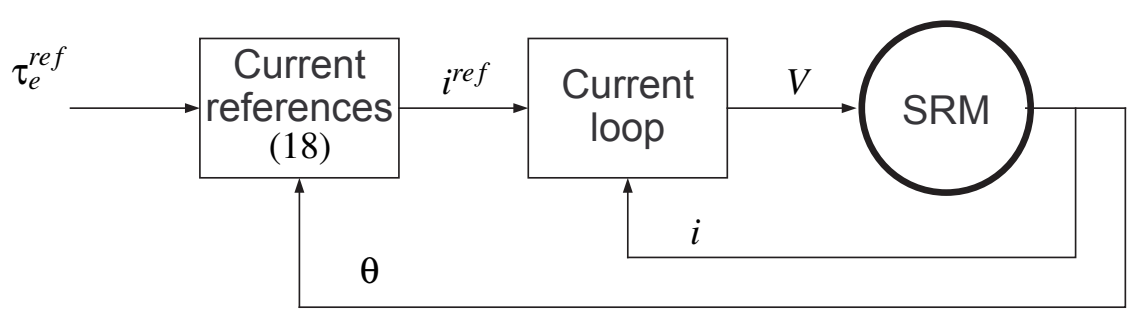

Figure 3: Proposed control scheme.

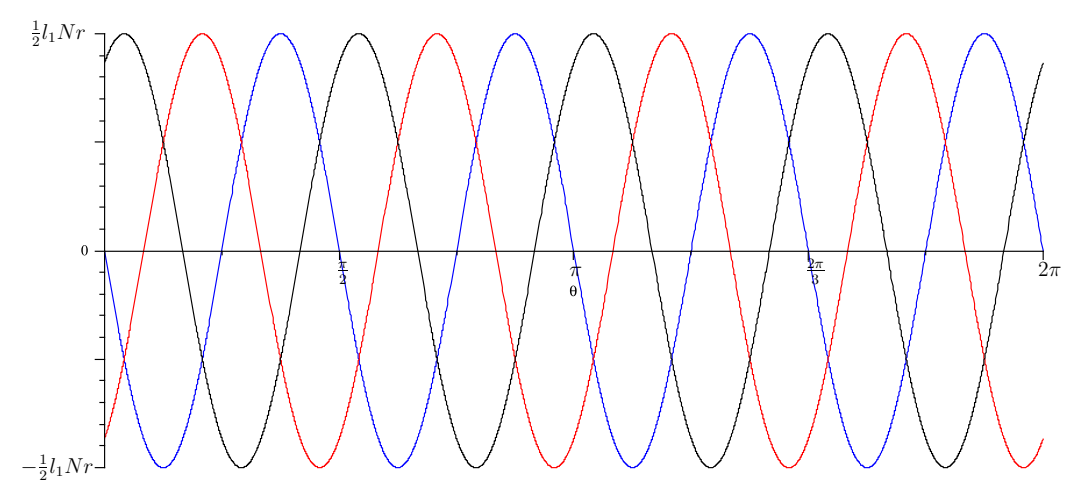

Figure 4: $-\partial_{\theta} L_{j}$ waveforms for a $6 / 4$ switched reluctance machine.

The main goal of this paper is to find the suitable current waveform such that the torque ripple is minimized. As a first approximation we will only consider the case that two phase cannot be voltage fed simultaneously. Tacking into account the last approximation, the torque ripple minimization has two steps: first, is to determine the angle range where each phase must be switch-on and, secondly, is to find the current waveform which ensures a minimum torque ripple.

\section{Ideal switch-on and switch-off angles}

From the model of a SRM presented in the previous Section, the torque equation (12) is the sum of sinusoidal waveforms, $-\partial_{\theta} L_{j}$, with the square of the currents, $i_{j}$. Notice that the current appears as $i_{j}^{2}$, and consequently, the produced torque does not depends on the sign of the phase current. It implies that the operation mode (generator or motor) only depends on the switch-on and switch-off angles. In Figure 4 is depicted the torque contribution of each phase without the current influence (or considering $i_{j}=1$ ).

Let us to consider that only one phase can be fed simultaneously. In this case, the appropriate range of the switch-on period, for each phase $j$, is around the angle were it corresponds the maximum value of $-\partial_{\theta} L_{j}$. This angle can be easily computed, differentiating (11), as

$$
\theta_{j}^{\max }=\frac{1}{N_{r}}\left(\frac{3 \pi}{2}-(j-1) \frac{2 \pi}{n}\right) .
$$

Then the preferred angle range while the $j$ phase is fed is when the $-\partial_{\theta} L_{j}$ function is bigger than $-\partial_{\theta} L_{(j-1)}$ and $\partial_{\theta} L_{(j+1)}$. This range values, $\theta_{j}^{\text {on }}$ and $\theta_{j}^{\text {off }}$, are defined by where

$$
\begin{aligned}
\theta_{j}^{\text {on }} & =\theta_{j}^{\max }-\frac{\pi}{n N_{r}} \\
\theta_{j}^{\text {off }} & =\theta_{j}^{\max }+\frac{\pi}{n N_{r}} .
\end{aligned}
$$

Figure 5 shows the $-\partial_{\theta} L_{j}$ waveform for a $6 / 4$ switched reluctance machine with $\theta_{j}^{\max }$ and the turn on and turn off angles, $\theta_{j}^{\text {on }}$ and $\theta_{j}^{\text {off }}$, respectively. 


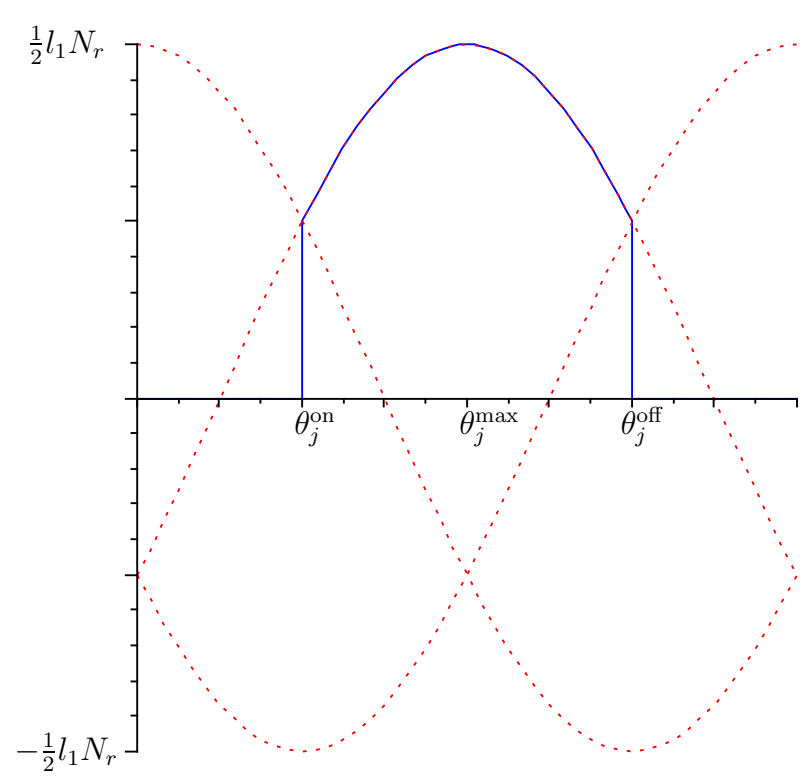

Figure 5: $-\partial_{\theta} L_{j}$ waveforms, and $\theta_{j}^{\max }, \theta_{j}^{\text {on }}$ and $\theta_{j}^{\text {off }}$ for a $6 / 4$ switched reluctance machine.

Tacking into account that, in an ideal case, only the $j$ current is different to zero while $\theta=\left(\theta_{j}^{\text {on }}, \theta_{j}^{\text {off }}\right)$, the following commutation policy is adopted

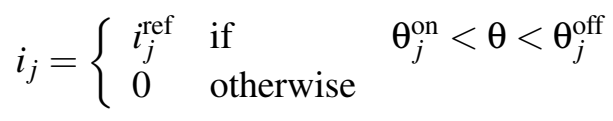

\section{Ideal current references}

Let us to determine the $j$ current references, $i_{j}^{\text {ref }}$. Assuming that the switching sequence is ideal (i.e., two consecutive phase turn on and turn off simultaneously), a constant instantaneous torque can be achieved. From (12) and replacing $\tau_{e}=\tau_{e}^{\text {ref }}$, the following references are obtained

$$
i_{j}^{\mathrm{ref}}=\sqrt{\frac{2 \tau_{e}^{\mathrm{ref}}}{l_{1} N_{r} \sin \left(N_{r} \theta+(j-1) \frac{2 \pi}{n}\right)}} .
$$

Figure 6 shows the $i_{j}^{\text {ref }}$ desired waveform for a 6/4 SRM machine.

\section{Simulations}

In this section we implement a numerical simulation of the proposed method (see Figure 3 ) for a $6 / 4$ switched reluctance machine. The inductance machine matrix corresponds to equation (8). In order to follow the current reference a PI controller is used for each phase. A synchronous centered-pulse singleupdate PWM strategy is used to implement the desired voltage, with a $5 \mathrm{kHz}$ switching frequency. The machine parameters are: $l_{0}=0.098 \mathrm{H}, l_{1}=0.082 \mathrm{H}, r=0.05 \Omega, N_{r}=4, b=0.005$ and $J=0.0001 \mathrm{~kg} \mathrm{~m}^{2}$. Figures 7 and 8 shows the phase currents obtained with the PI controller. Note that, in Figure 7, the real and desired currents for the a-phase, $i_{a}$ and $i_{a}^{r e f}$, differs due to the electrical dynamic of each inductance.

Figure 9 compares the produced torque with the torque minimization approach and a constant current reference. The simulation results show that the proposed current references reduces the torque ripple.

Oscillations in the torque are due to the fact that the current control does not achieve perfectly the proposed reference, see Figure 7. Advanced control techniques for the current loop control, such as adaptive methods or nonlinear controllers (for example sliding mode techniques), could improve the performance. 


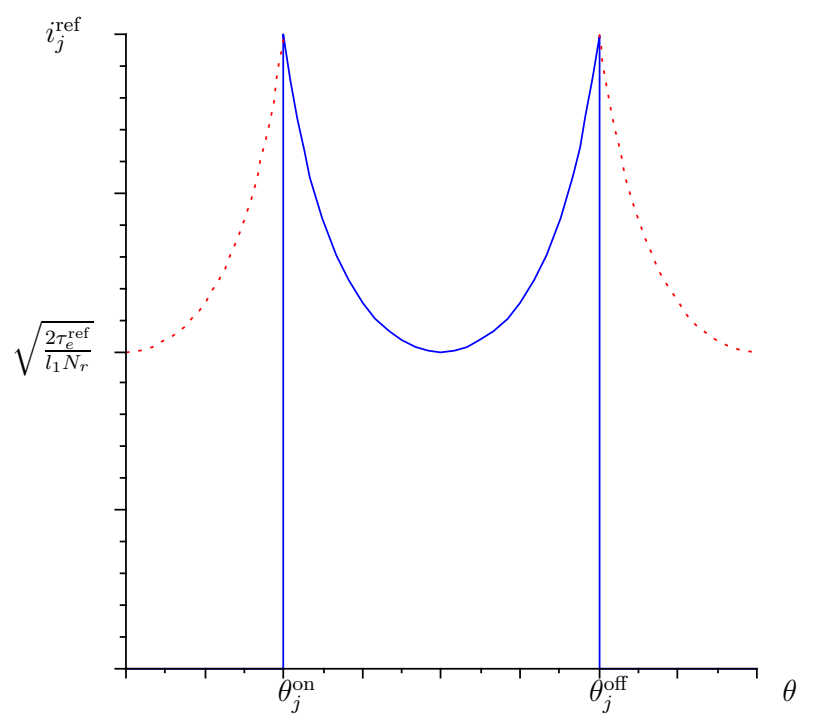

Figure 6: $i_{j}^{\text {ref }}$ waveform for a 6/4 switched reluctance machine.

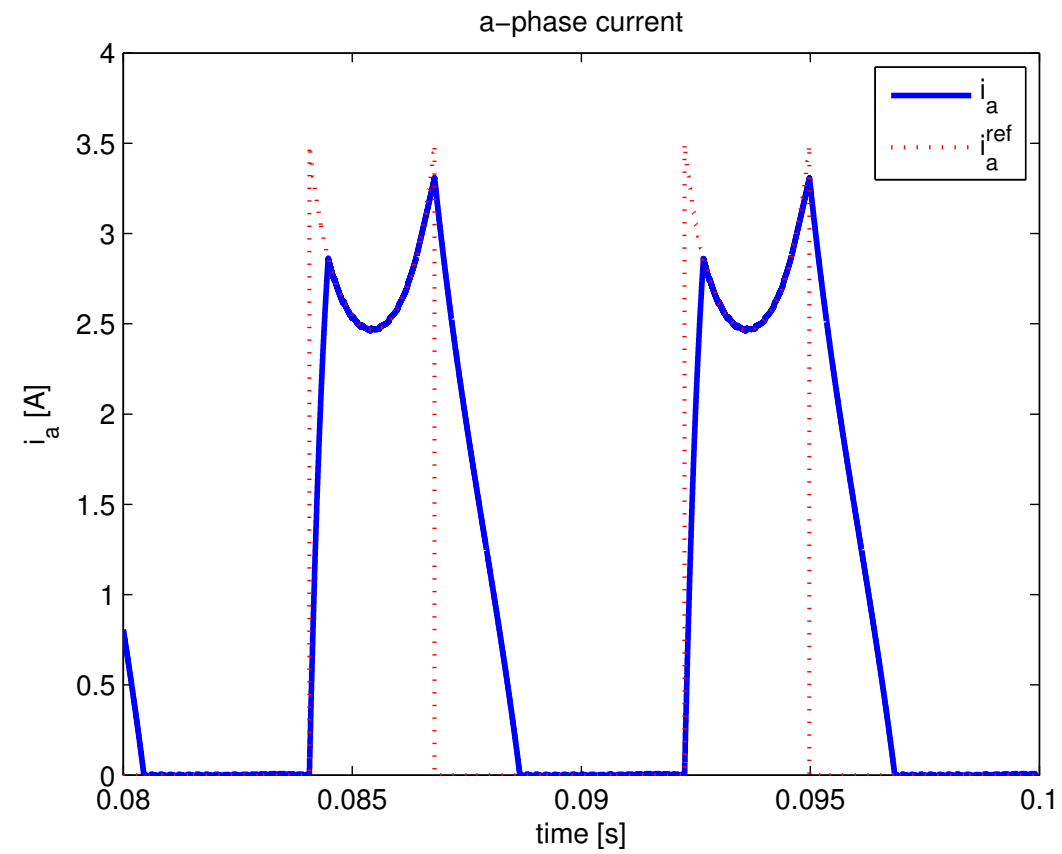

Figure 7: $a$-phase current and its desired value $i_{a}^{\text {ref }}$ for a $6 / 4$ switched reluctance machine with the torque ripple minimization method. 


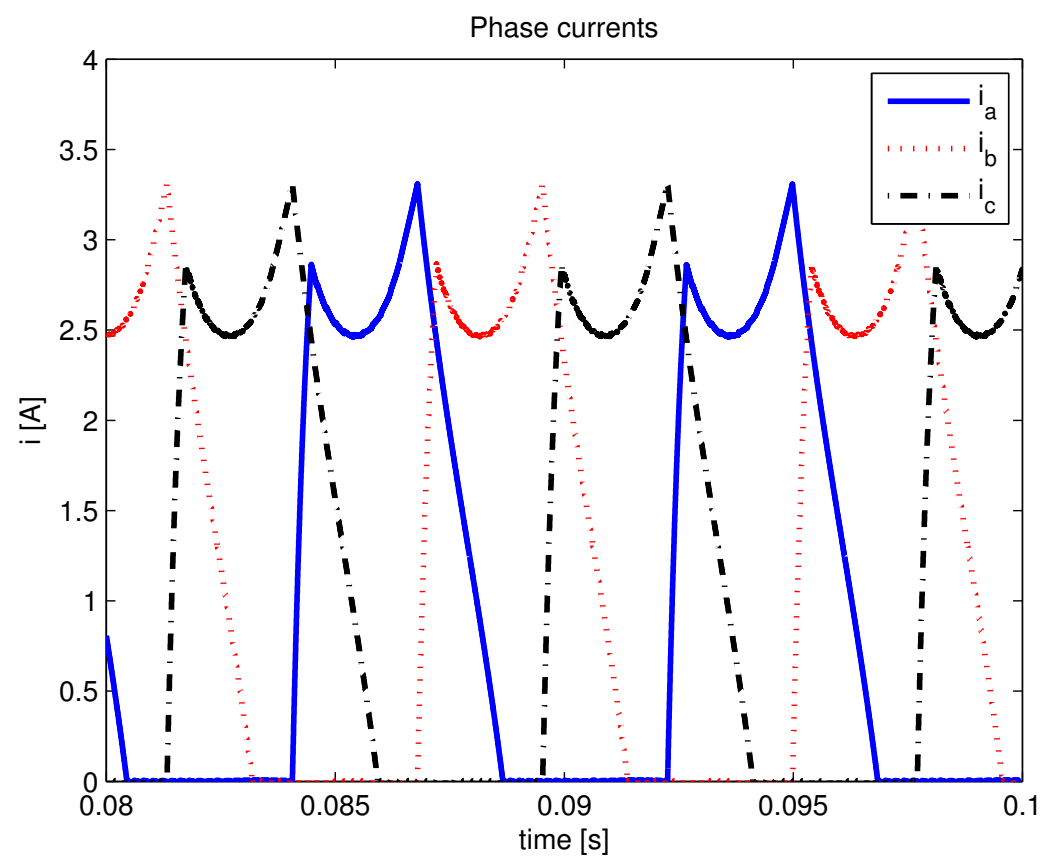

Figure 8: Current waveform for a 6/4 switched reluctance machine with the torque ripple minimization method.

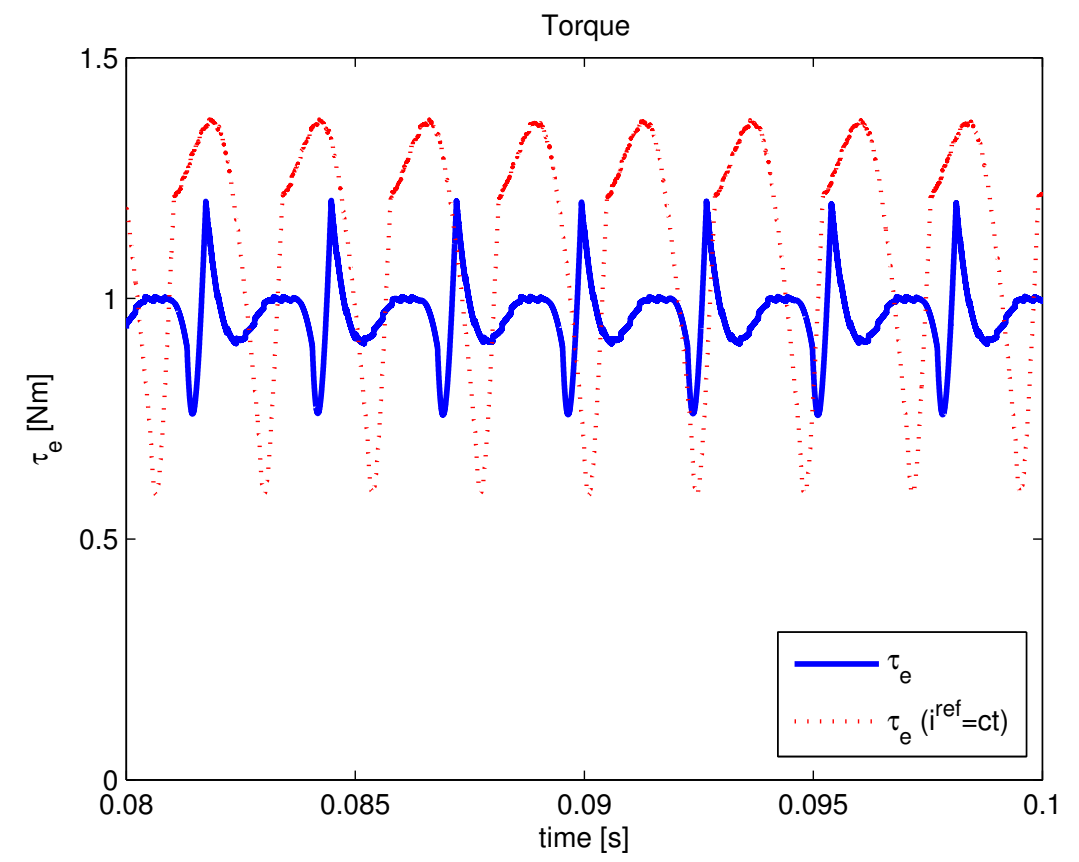

Figure 9: Comparison of the produced torque using the proposed method and a constant $i_{j}^{\text {ref }}$ for a $6 / 4$ switched reluctance machine. 


\section{Conclusions}

In this paper the torque ripple minimization is studied. From the torque equation, and evaluating the optimal switch on angle for each phase, a current reference is proposed in order to minimize the torque ripple. The proposed method is validated via simulation, using standard PI controllers for the current loops, and also compared with the constant current reference case.

The paper is focused in a first harmonic approximation but higher harmonics cases can be easily also studied. More complicate scenario is including mutual inductances or saturation effects, appearing cross and current depending terms in the $\mathcal{L}$ matrix. All these cases can be also treated in the future.

\section{References}

[1] I. Husain and M. Ehsani. Torque ripple minimisation in switched reluctance motor drives by pwm current control. IEEE Trans. on Power Electronics, 11(1):83-88, 1996.

[2] N. Inanc and V. Ozbulur. Torque ripple minimization of a switched reluctance motor by using continous sliding mode control technique. Electric Power System Research, 66:241-251, 2003.

[3] R.B. Inderka, M. Menne, and R.W.A.A De Doncker. Control of switched reluctance drives for electric vehicle applications. IEEE Trans. on Industrial Electronics, 49(1):48-53, 2002.

[4] R. Krishnan. Switched Reluctance Motor Drives. CRC Press, 2001.

[5] T.J.E Miller. Switched Reluctance Motor and their control. Clarendon-Oxford, 1993.

[6] K.M. Rahman, B. Fahimi, G. Suresh, A.V. Rajarathnam, and M. Ehsani. Advantages of switched reluctance motor applications to ev and hev: design and control issues. IEEE Trans. on Industry Applications, 36(1):111-121, 2000.

[7] Y. Sozer and D.A. Torrey. Optimal turn-off angle control in the fac of automatic turn-on angle control for switched-reluctance motors. IET Electric Power Applications, 1(3):395-401, 2007. 